University of Nebraska - Lincoln

DigitalCommons@University of Nebraska - Lincoln

$12-14-2003$

\title{
Strong field detachment of a negative ion with non-zero angular momentum: application to $\mathrm{F}^{-}$
}

\author{
M. V. Frolov \\ University of Nebraska - Lincoln \\ N. L. Manakov \\ Voronezh State University, manakov@phys.vsu.ru \\ E. A. Pronin \\ Voronezh State University, Voronezh 394006, Russia \\ Anthony F. Starace \\ University of Nebraska-Lincoln, astarace1@unl.edu
}

Follow this and additional works at: https://digitalcommons.unl.edu/physicsstarace

Part of the Physics Commons

Frolov, M. V.; Manakov, N. L.; Pronin, E. A.; and Starace, Anthony F., "Strong field detachment of a negative ion with non-zero angular momentum: application to $\mathrm{F}^{-1}$ (2003). Anthony F. Starace Publications. 138. https://digitalcommons.unl.edu/physicsstarace/138

This Article is brought to you for free and open access by the Research Papers in Physics and Astronomy at DigitalCommons@University of Nebraska - Lincoln. It has been accepted for inclusion in Anthony F. Starace Publications by an authorized administrator of DigitalCommons@University of Nebraska - Lincoln. 
Published in Journal of Physics B: Atomic, Molecular and Optical Physics 36:23 (December 14, 2003), pp. L419L426. Copyright $@ 2003$ IOP Publishing Ltd. Used by permission.

http://stacks.iop.org/IPhysB/36/L419

Submitted August 20, 2003; published online November 11, 2003.

LETTER TO THE EDITOR

\title{
Strong field detachment of a negative ion with non-zero angular momentum: application to $\mathrm{F}^{-}$
}

\author{
M. V. Frolov ${ }^{1,2}$, N. L. Manakov², E. A. Pronin ${ }^{2}$, and Anthony F. Starace ${ }^{1}$ \\ ${ }^{1}$ Department of Physics and Astronomy, University of Nebraska-Lincoln, Lincoln, NE 68588-0111, \\ USA \\ ${ }^{2}$ Physics Department, Voronezh State University, Voronezh 394006, Russia
}

\begin{abstract}
We apply our recently developed, model-independent quantum approach for intense laser detachment of a weakly bound electron to interpret a recent experiment on abovethreshold detachment (ATD) of the $\mathrm{F}^{-}$ion. We find that the measured electron energies correspond to the "Keldysh part" of the ATD spectrum, just below the onset of our predicted rescattering plateau. Overall, our predicted ATD spectrum (using a scaled peak intensity and focal averaging) is in excellent agreement with the experimental data, except for certain structures observed for electron energies above $12.6 \mathrm{eV}$ that we attribute to known two-electron resonances of $\mathrm{F}^{-}$. A number of analytical approximations to our exact p-state ATD amplitude are obtained, and their accuracy is investigated.
\end{abstract}

There are only a few analytical models that are widely used in laser-atom physics to describe strong field ionization and detachment processes. The simplest of them is the Keldysh approximation (KA) [1], which is valid for the tunneling regime. The most spectacular feature in ATI and ATD spectra, the broad plateau in the yield of high-energy electrons, originates from the rescattering of the active electron by the parent ion or atom. It requires for its description a more exact, beyond-KA account of the binding potential $U(r)$. A few such treatments have been introduced. An "improved" version of the KA (IKA) [2], that includes first-order in $U(r)$ corrections to the KA, has been used to interpret experiments for inert gases, as reviewed in [3]. Quasiclassical analyses of rescattering effects have become popular recently; they interpret high-energy plateau features in terms of classical trajectories [3]. Finally, a fruitful model for analyzing strong field effects beyond the KA is the zero-range potential (ZRP) model, which (within the quasistationary quasienergy states (QQES) approach [4]) permits an accurate, ab initio formulation of the problem as well as essentially exact numerical results $[5,6]$. However, the above mentioned beyond-KA analyses are valid only for s-electron initial states and thus do not allow one to analyze the dependence of ATI or ATD features on the spatial symmetry of the initial state. Very recently [7], a model-independent approach has been developed for strong field detachment of a weakly bound electron having 
a non-zero angular momentum. It predicts a number of distinctive features in strong field ATD of negative ions with outer s and p electrons. Also recently [8], the first experiment on ATD with absorption of many (up to 23) excess photons was performed for the $\mathrm{F}^{-}$ion. Reference [8] interprets the experimental p-state data using a KA analysis [9], and rules out the rescattering mechanism as irrelevant (owing to the small magnitude of the plateau predicted for s-state ions).

In this letter, we relate our model-independent approach [7] for p-state ions to the KA and beyond-KA analyses, present simple analytical approximations to our exact results, and use our approach to interpret the experimental results of [8].

The key idea of our approach [7] is to solve the exact Schrödinger equation for the complex quasienergy, $\epsilon$, and the QQES wavefunction, $\Phi \epsilon(\mathbf{r}, t)$, for an electron in a finite-range potential, $U(r)$, subjected to a strong linearly polarized laser field, $\mathbf{F}(t)=\mathbf{F} \cos (\omega t)$, by proper matching of solutions inside the potential well, $U(r)$, with those appropriate at large distances, where the effects of $U(r)$ are negligible. Thus the general expression for $\Phi_{\epsilon}(\mathbf{r}, t)$ may be represented as a wavepacket of free electron states in a laser field $\mathbf{F}(t)$. For a short-range potential, $U(r)$, that supports a shallow bound state, $\psi_{\text {кlm }}{ }^{(0)}(\mathbf{r})$, having an energy $E_{0}=-\left(\hbar^{2} \kappa^{2}\right) / 2 m$ and an angular momentum $l$ (i.e. $U(r)=0$ for $r>r_{c^{\prime}}$ where $\kappa r_{c} \ll 1$ ), the key simplification in this matching is that the solution at $0 \ll r \ll \kappa^{-}$ 1 is independent of the shape of $U(r)$. It has the following form [7] (cf. similar analyses [10, 11] for time-independent Hamiltonians):

$$
\Phi_{\epsilon}(\mathbf{r}, t) \sim Y_{l m}(\hat{r}) \sum_{s}\left[r^{-l-1}+\cdots+r^{l} B_{l}(\epsilon+s \hbar \omega)\right] f_{s} \exp (-\mathrm{i} s \omega t),
$$

where the $f_{s}$ are Fourier coefficients of a periodic function $f_{\epsilon}(t)=\Sigma_{s} f_{s} \exp (-\mathrm{i} s \omega t)$, and where the effective range parameterization [12],

$$
(2 l-1) ! !(2 l+1) ! ! B_{l}(E)=-1 / a_{l}+r_{l} k^{2} / 2, \quad k^{2}=2 m E / \hbar^{2},
$$

is used for the mixing coefficient $B_{l}$ between the regular $\left(\sim r^{l}\right)$ and irregular $\left(\sim r^{-l-1}\right)$ solutions of the unperturbed Schrödinger equation at $r_{\mathrm{c}}<r \ll \kappa^{-1}$ [12]. The physical meaning of equation (1) is that for small $r$ the main effects of the laser field are

(i) to change the relation between regular and irregular solutions for angular momentum $l$ (due to dependence of the coefficient $B_{l}$ in (1) on the laser parameters), and

(ii) to introduce harmonic time-dependent factors. It implies also that inside the well other (laser-induced) angular momentum components are negligibly small.

This form is valid for laser field amplitudes $F$ such that $\left|\left(\epsilon(F)-E_{0}\right) / E_{0}\right| \ll 1$. (For the peak laser intensities considered in our calculations, this ratio is of order $10^{-3}$.) Outside the well, of course, laser-induced angular momentum components with $l^{\prime} \geq|m|$ are very important and are included in our treatment (see [7]). For our case of an initial bound state, $\psi_{\text {klm }}{ }^{(0)}(\mathbf{r})$, having the known asymptotic form at $r \rightarrow \infty$,

$$
\psi_{\kappa l m}^{(0)}(\mathbf{r})=R_{\kappa l}^{(0)}(r) Y_{l m}(\hat{r}), \quad R_{\kappa l}^{(0)}\left(r \gg \kappa^{-1}\right) \approx C_{l} r^{-1} \exp (-\kappa r),
$$

the fundamental parameters of the effective range approach for collisional problems, the scattering length $\left(a_{l}\right)$ and the effective range $\left(r_{l}\right)$, may be expressed in terms of the binding energy (or $\kappa=$ $\left.\left(2 m\left|E_{0}\right|\right)^{1 / 2} / \hbar\right)$ and the asymptotic coefficient $C_{l}$ in (2) [13]:

$$
(-1)^{l} \kappa^{2 l+1}-a_{l}^{-1}-r_{l} \kappa^{2} / 2=0, \quad(-1)^{l}(2 l+1)-r_{l} \kappa^{-(2 l-1)}=2 \kappa C_{l}^{-2} .
$$

Thus, in our analysis we regard $E_{0}$ and $C_{l}$ as parameters of the problem.

The important difference from similar analyses of quasistationary states of a weakly bound electron subjected to static perturbations $[10,11]$ is that in our time-dependent case the asymptotic form (1) of the QQES wavefunction at small $r$ is time dependent, as it should be in accord with 
the general (Floquet) form of a quasienergy solution. The function $f_{\epsilon}(t)$ enters the counterpart of the solution (1) outside the well $U(r)$ (at $r>r_{c}$ ), which has an outgoing-wave asymptotic form at $r \rightarrow \infty$ and the singularity $\sim r^{-l-1}$ (as in (1)) at small $r$ [7]. For a given $l$, this solution may be presented in terms of the retarded Green function for a free electron in the laser field $\mathbf{F}(t)$ (see [7] for details). The matching of in-well and out-of-well solutions yields the key result of our approach, an integro-differential eigenvalue equation for $\epsilon$ and the periodic function $f_{\epsilon}(t)$ that is equivalent to an infinite system of linear homogeneous equations for the even Fourier coefficients, $f_{s=2 k}$ [7]. For an initial $\mathrm{p}$ state, this system has the following form in scaled units (in which energies and $\hbar \omega$ are measured in units of $\left|E_{0}\right|$, and the field amplitude $F$ in units of $\left.F_{0}=\left(2 m\left|E_{0}\right|^{3}\right)^{1 / 2} /|e| \hbar\right)$ :

$$
\left[1-(-\epsilon-2 k \omega)^{3 / 2}+\frac{r_{1}}{2}(1+\epsilon+2 k \omega)\right] f_{2 k}=\sum_{k^{\prime}}\left[M_{k, k^{\prime}}(\epsilon)+\delta_{m, 0} \tilde{M}_{k, k^{\prime}}(\epsilon)\right] f_{2 k^{\prime}} .
$$

The explicit form of the matrix elements in terms of Bessel functions $J_{\mathrm{p}}(x)$ is

$$
\begin{aligned}
M_{k, k^{\prime}}(\epsilon)=- & \frac{3}{4} \sqrt{\frac{\mathrm{i}}{\pi}} \mathrm{i}^{k-k^{\prime}} \int_{0}^{\infty} \frac{\mathrm{e}^{\mathrm{i}\left(\epsilon+\left(k+k^{\prime}\right) \omega\right) \tau}}{\tau^{5 / 2}}\left[\mathrm{e}^{-\mathrm{i} \lambda(\tau)} J_{k-k^{\prime}}(z(\tau))-\delta_{k, k^{\prime}}\right] \mathrm{d} \tau, \\
\tilde{M}_{k, k^{\prime}}(\epsilon)=- & \frac{3}{4} \sqrt{\frac{\mathrm{i}}{\pi}} \mathrm{i}^{k-k^{\prime}}\left(\frac{F}{\omega}\right)^{2} \int_{0}^{\infty} \frac{\mathrm{e}^{\mathrm{i}\left(\epsilon+\left(k+k^{\prime}\right) \omega\right) \tau-\mathrm{i} \lambda(\tau)}}{\tau^{3 / 2}} \\
& \times\left[J_{k-k^{\prime}}(z(\tau)) j_{1}(\tau)+\mathrm{i} J_{k-k^{\prime}}^{\prime}(z(\tau)) j_{0}(\tau)\right] \mathrm{d} \tau,
\end{aligned}
$$

where the following notations are used:

$$
\begin{aligned}
& \lambda(\tau)=\frac{u_{\mathrm{p}}}{\omega}\left(\omega \tau-\frac{4 \sin ^{2} \omega \tau / 2}{\omega \tau}\right), \quad z(\tau)=\frac{u_{\mathrm{p}}}{\omega}\left(\sin \omega \tau-\frac{4 \sin ^{2} \omega \tau / 2}{\omega \tau}\right), \\
& j_{v}(\tau)=\frac{\sin ^{2} \omega \tau / 2}{(\omega \tau / 2)^{2}}-2 \frac{\sin \omega \tau}{\omega \tau}+\cos ^{\nu} \omega \tau, \quad v=0,1,
\end{aligned}
$$

where $u_{\mathrm{p}}=F^{2} /\left(2 \omega^{2}\right)$ is the ponderomotive shift $U_{\mathrm{p}}$ in units of $\left|E_{0}\right|$. The matrix elements (4) and (5) are easily calculated numerically by the method described in [6] for a ZRP model, thus providing exact numerical results for $\epsilon$ and $f_{2 k}$.

With known $\epsilon$ and the set of coefficients $f_{2 k^{\prime}}$ the $n$-photon ATD amplitude, $\mathcal{A}_{n}^{(m)}(\mathbf{n})$, for electron ejection in the direction $\mathbf{n}$, is given by the $n$th Fourier coefficient of $\Phi_{\epsilon}(\mathbf{r}, t)$ at $r \rightarrow \infty$ [7]. For a $\mathrm{p}$ state, the result is

$$
\begin{aligned}
& \mathcal{A}_{n}^{(m)}(\mathbf{n})=\sum_{k=-\infty}^{\infty}(-1)^{k} f_{2 k} \mathcal{A}_{n k}^{(m)}(\mathbf{n}), \\
& \mathcal{A}_{n k}^{(m)}(\mathbf{n})=C_{1}\left[k_{n} Y_{1, m}(\mathbf{n}) \mathcal{J}_{0}(n-2 k, \alpha, \beta)-\delta_{m, 0} \sqrt{\frac{3}{4 \pi}} \frac{F}{\omega} \mathcal{J}_{1}(n-2 k, \alpha, \beta)\right],
\end{aligned}
$$

where $\alpha=2 F k_{n} \cos \theta / \omega^{2}, \beta=u_{\mathrm{p}} /(2 \omega), \theta$ is the angle between $\mathbf{n}$ and $\mathbf{F}, k_{n}=\left(\epsilon+n \omega-u_{\mathrm{p}}\right)^{1 / 2}$, and $\mathcal{J}_{m}(s, \alpha$, $\beta$ ) is a generalized Bessel function [14], which satisfies the following relations:

$$
\mathcal{J}_{0}(s, \alpha, \beta)=\sum_{\mathrm{p}} J_{s+2 p}(\alpha) J_{\mathrm{p}}(\beta), \quad 2 \mathcal{J}_{1}(s, \alpha, \beta)=\mathcal{J}_{0}(s+1, \alpha, \beta)+\mathcal{J}_{0}(s-1, \alpha, \beta) .
$$

As is obvious from (6) and (7), the $n$-photon differential detachment rate depends only on the modulus of the angular momentum projection, $m$, of an initial $\mathrm{p}$ state, which is a conserved quantum number in the presence of a linearly polarized field:

$$
\frac{\mathrm{d} \Gamma_{n}^{(|m|)}(\theta)}{\mathrm{d} \Omega}=2\left|\sqrt{k_{n}} \mathcal{A}_{n}^{(m)}(\mathbf{n})\right|^{2} .
$$


The exact results (6)-(8) have a transparent structure that allows one to relate them to previous studies as well as to obtain simple beyond-KA approximations.

(i) Relation to the ZRP model. Applying our approach to an initial s state, instead of (3) we obtain for $\epsilon$ and $f_{2 k}$ a system of equations involving $r_{0}=\kappa^{-1}-2 C_{0}^{-2}$. This system coincides with that for a ZRP model $[4,6]$ after replacing $r_{0}$ by zero and $C_{0}$ by $C_{0}^{\mathrm{ZRP}}=(2 \kappa)^{1 / 2}$. This result is expected, since the bound state $\psi_{0}(r)$ for a ZRP has the form (2) (with $l=0$ ) for any $r$; thus, in the limit $r_{0} \rightarrow 0$, matching of in-well and out-of-well solutions may be replaced by the well known boundary condition for $\Phi_{\epsilon}(\mathbf{r}, t)$ at the origin. Therefore, even for s states our modelindependent approach is somewhat more general than the ZRP model, giving the effectiverange-based justification for introducing the asymptotic factor $C_{0}$ when applying the ZRP results to ATD of real ions, such as, e.g., $\mathrm{H}^{-}$(cf. [15] for the case of photodetachment).

(ii) The KA limit. As discussed in [7], our approach encompasses the KA result as a limiting case when $\epsilon=E_{0}=-1$ and $f_{2 k}=\delta_{k, 0}$. Thus, the "exact" KA amplitude (in terms of generalized Bessel functions, i.e., without using a low frequency expansion) is given by $\mathcal{A}_{n 0}^{(m)}(\mathbf{n})$ in (7). Numerically, our KA results for $\omega<0.3$ agree very well with those of the approximate KA analysis of [9], which in our approach corresponds to a saddle-point analysis of the one-dimensional integral representation for the generalized Bessel functions.

(iii) The first-order rescattering approximation ( $R A)$. The terms (7) with $\mathrm{k} \neq 0$ describe binding potential (or, in quasiclassical terminology, rescattering) effects in the ATD amplitude (6) that are neglected in the KA. Their "intensity" is given by the coefficients $f_{2 k^{\prime}}$ which define the QQES wavefunction (1) in the small- $r$ region (where binding potential effects are important) and which exhibit a plateau-like behavior as a function of $k$ [7]. Thus, the exact dynamics of laseratom interactions are described by a complicated homogeneous system of equations (3) for the $f_{2 k}$ coefficients and the eigenvalue $\epsilon$. Any iterative (in $U(r)$ ) analysis should be done carefully; indeed, such an iterative procedure is similar to a Born expansion for a bound state, the accuracy of which is rather unclear. To find the first-order in $U(r)$ correction to the KA result, we put all diagonal matrix elements in equation (3) on the left-hand side and then substitute the KA result for $f_{2 k}$ (i.e. $f_{2 k}=\delta_{k, 0}$ ) on the right-hand side. The RA result for the coefficients $f_{2 k}$ is thus

$$
f_{2 k}^{\mathrm{RA}}=\frac{M_{k, 0}(-1)+\delta_{|m|, 0} \tilde{M}_{k, 0}(-1)}{1-(1-2 k \omega)^{3 / 2}+r_{1} k \omega-M_{k, k}(-1)-\delta_{|m|, 0} \tilde{M}_{k, k}(-1)}, \quad k \neq 0 .
$$

Note that the form of our RA result (9) for p states is identical to the corresponding RA result for s states in a ZRP model (cf. approximation I in [6]).

(iv) The IKA result for $p$ states. To obtain a generalization of the IKA result $[2,3]$ for $\mathrm{p}$ states, we note that for s states it follows as a simplified version of the RA, replacing the denominator in the equation similar to (9) by unity (see approximation II in [6]). In a similar way, we introduce the IKA for $\mathrm{p}$ states as follows:

$$
f_{2 k}^{\mathrm{IKA}}=M_{k, 0}(-1)+\delta_{|m|, 0} \tilde{M}_{k, 0}(-1), \quad k \neq 0, \quad f_{0}=1 .
$$

Although this approximation has no theoretical justification (based upon the exact equation (3)), the numerical results presented below show that its predictions differ only slightly from those of the RA in the region beyond the plateau onset.

To demonstrate the dependence of the ATD spectrum of $p$ electrons in the strong field regime on the frequency, $\omega$, and to verify the accuracy of approximations (ii)-(iv), in figure 1 we pres- 

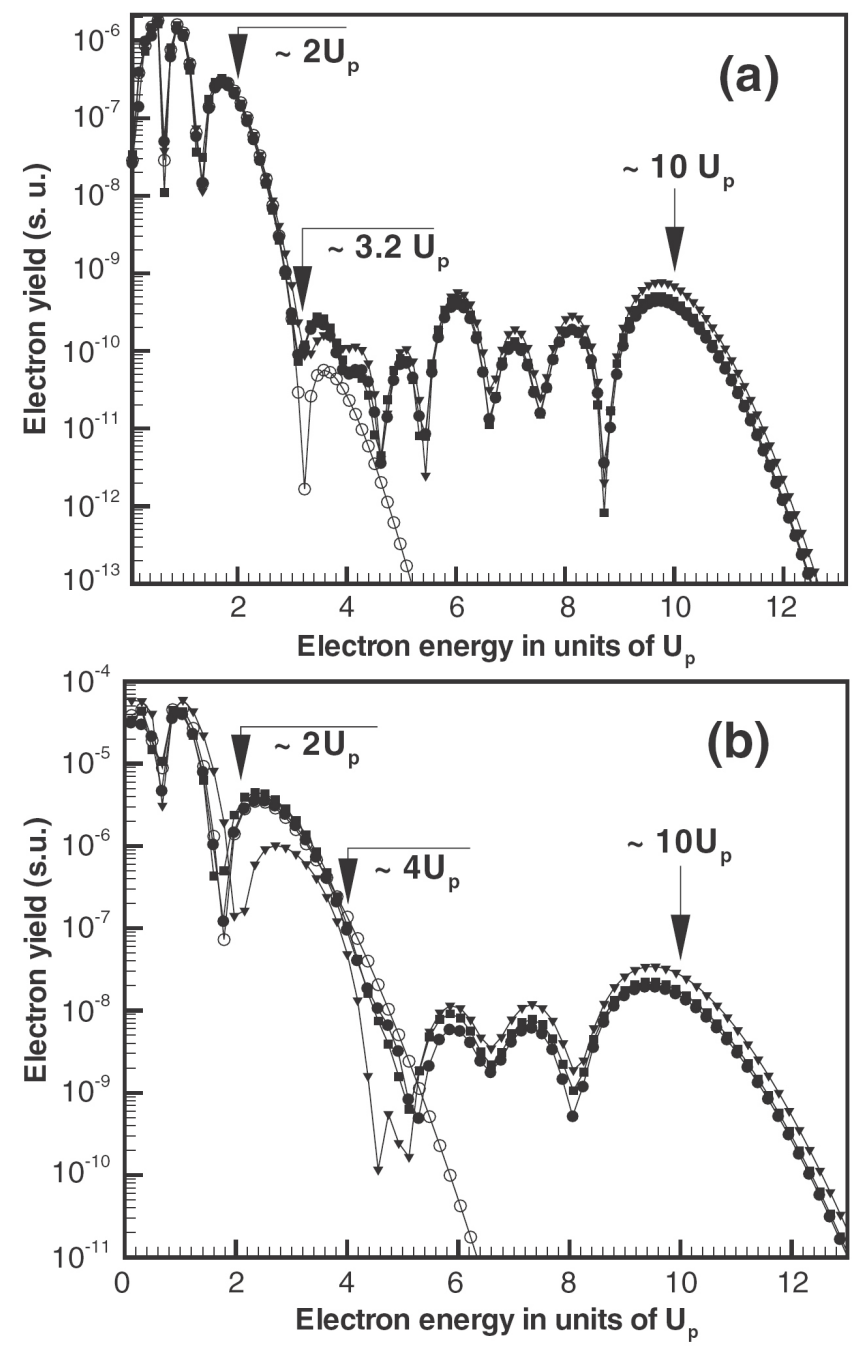

Figure 1. The ATD spectrum (in scaled units) of $\mathrm{F}^{-}$along the laser polarization axis $(\theta=0)$ : (a) $\omega=0.128, F=$ 0.1895 ; (b) $\omega=0.2026, F=0.3$. Full circles = exact results according to equations (3) and (6)-(8); full squares $=$ the RA (9); full triangles = the IKA (10); open circles = the KA result. Lines connecting the symbols are added for better visualization. The arrows indicate the "classical" plateau onset at $2 U_{p^{\prime}}$ the cut-off at $10 U_{p^{\prime}}$ and the KA breakdown points.

ent ATD spectra (averaged over the projection $m$ ) for two different sets of $\omega$ and field amplitude, $F$, keeping the same value for the Keldysh parameter, $\gamma=\omega / F=0.675$, or $u_{\mathrm{p}}=1 /\left(2 \gamma^{2}\right)=1.097$. The parameters $E_{0}$ and $C_{1}$ were chosen for the $\mathrm{F}^{-}$ion: $\left|E_{0}\right|=3.4 \mathrm{eV}$ and $C_{1}=0.84$ au. Thus, for $\mathrm{F}^{-}$, the scaled field amplitude, $F_{0^{\prime}}$ and the scaled intensity, $I_{0}=c F_{0}^{2} /(4 \pi)$, are $F_{F}{ }^{-}=2.01 \times 10^{7} \mathrm{~V} \mathrm{~cm}^{-1}$ and $I_{F}$ $=1.37 \times 10^{14} \mathrm{~W} \mathrm{~cm}^{-2}$.

One sees that the difference between our KA and our exact results becomes smaller as the frequency decreases. The onset of the high energy part of the ATD spectrum (where the KA breaks down) depends significantly on both the frequency and the Keldysh parameter. Nevertheless, we find that the plateau onset position is more sensitive to $\omega$ than to $\gamma$. For instance, at the same $\gamma$, but for different frequencies (as in figure 1), the onset of the plateau for smaller frequency appears before that for higher frequency (i.e., at $3.2 U_{\mathrm{p}}$ versus $\sim 5 U_{\mathrm{p}}$ ). Increasing the intensity (or decreasing $\gamma$ ) at fixed $\omega$ moves the onset closer to the classical estimate, $E \approx 2 u_{\mathrm{p}}$ [3]. Thus, the classical on- 
set position corresponds to the case of extremely small frequencies $(\omega<0.1)$ and relatively high intensities, for which $\gamma \leq 0.5$. As far as we know, only an experiment for He [16] clearly indicates the plateau onset at $E \approx 2 u_{\mathrm{p}}$; in many experiments for other inert gases (see, e.g., [17]), the onset position was found to "float" in the interval between $\sim 3 u_{\mathrm{p}}$ and $\sim 5 u_{\mathrm{p}}$.

Concerning the accuracy of the RA and IKA approximations, figure 1(a) shows that for $\omega$ $=0.128$ results of both approximations are in good agreement with the exact results. However, for $\omega=0.203$, figure $1(\mathrm{~b})$ shows that the IKA surprisingly fails for low energies, whereas the KA works well. We conclude that with increasing frequency the binding potential effects become relatively more important. Therefore, the neglect of the denominator in equation (9), which involves the binding potential through the diagonal matrix elements $M_{k, k}$ and $\hat{M}_{k, k^{\prime}}$ can dramatically affect the photoelectron yield in the low energy part of the ATD spectrum, where strong interference between the (KA) amplitude for direct detachment and the "rescattering" amplitudes with $k \neq 0$ in equation (6) may occur. In the low frequency case, the denominator in equation (9) is a smooth function of $k$, and can be replaced by a constant. In some sense, this fact justifies the IKA for $\omega \ll 1$.

In order to compare our predictions to the recent experiment for $\mathrm{F}^{-}$[8], we have performed a spatial averaging of our results over the Gaussian shape of the laser pulse with the same focal parameters as in [8]. To simplify the calculations, we used the RA result (9) for $f_{2 k}$ to calculate the ATD spectra for a range of intensities. We chose the peak laser intensity to fit our theoretical predictions to the experimental data for slow electrons (up to $5 \mathrm{eV}$ ). We find that the best average agreement corresponds to a peak laser intensity of $1.6 \times I_{\text {exp }}$, where $I_{\exp }=1.1 \times 10^{13} \mathrm{~W} \mathrm{~cm}^{-2}$ is the peak intensity estimated in [8]. An underestimation of the peak intensity may be caused by the difficulties inherent to defining the size of the interaction region and the focal parameters of the laser beam, which are fitted by a Gaussian distribution. (Note that to clarify this problem [18] introduced a "fractional shift", which is the ratio between the experimental and theoretical intensities. It was shown that this ratio ranges from 0.5 to 2.) As seen in figure 2(a), for slow electrons, up to $\sim 12.6 \mathrm{eV}$, the experimental data are described very well by our predictions. Nevertheless, despite the fact that the experimental measurements have been made for relatively small energies, where high order binding potential effects seem not to be important and where the KA should work well, we observe significant differences between our results and the experimental data for energies of $\sim(12.6-15.4)$ and $\sim 17.2 \mathrm{eV}$. The neutral $\mathrm{F}$ atom has a series of excited $n=3$ levels in the range $12.7-16.0 \mathrm{eV}$ as well as one at $17.2 \mathrm{eV}$, just below the ionization limit at $17.4 \mathrm{eV}$ [19]. ATD electrons with energies in these regions may resonantly excite these F atom levels [20]. We postulate that the series of peaks in the experimental ATD spectrum shown in figure 2(a) may originate from such core-excited shape resonances, i.e., the active ATD electron may scatter from the F atom core to form doubly excited states of $\mathrm{F}^{-}$, which decay to the ground and excited states of $\mathrm{F}$. As predicted in [21] (see Table XVIII there), the recommended (theoretical) positions of such resonances are at 12.29, 14.85, and $17.69 \mathrm{eV}$, which are close to the observed structures in figure 2(a). The width of these shape resonances is much higher than the width of Feshbach resonances. Thus, the experimental resonant structures may be attributed to overlapping shape resonances. In figure 2(b) we present a 3D overview of the photoelectron yield (after focal averaging) as a function of both the electron energy and the ejection angle $\theta$ (see also the inset in figure 2(a) for $\theta=0$ ). For slow electrons (up to $12 \mathrm{eV}$ ) this picture is similar to that obtained experimentally and fitted by KA results [9] (see Figure 1 in [8]). For higher electron energies, the ATD plateau becomes localized about $\theta=0$ and drops sharply with increasing $\theta$. The pattern of oscillation with electron energy and angle $\theta$, which is very pronounced in the 3D results for a fixed intensity [7], is smoothed by focal averaging, but is still visible.

In conclusion, in this letter we have applied our new theoretical formulation (for describing a weakly bound electron having angular momentum $l=1$ in an intense laser field) to interpret the 

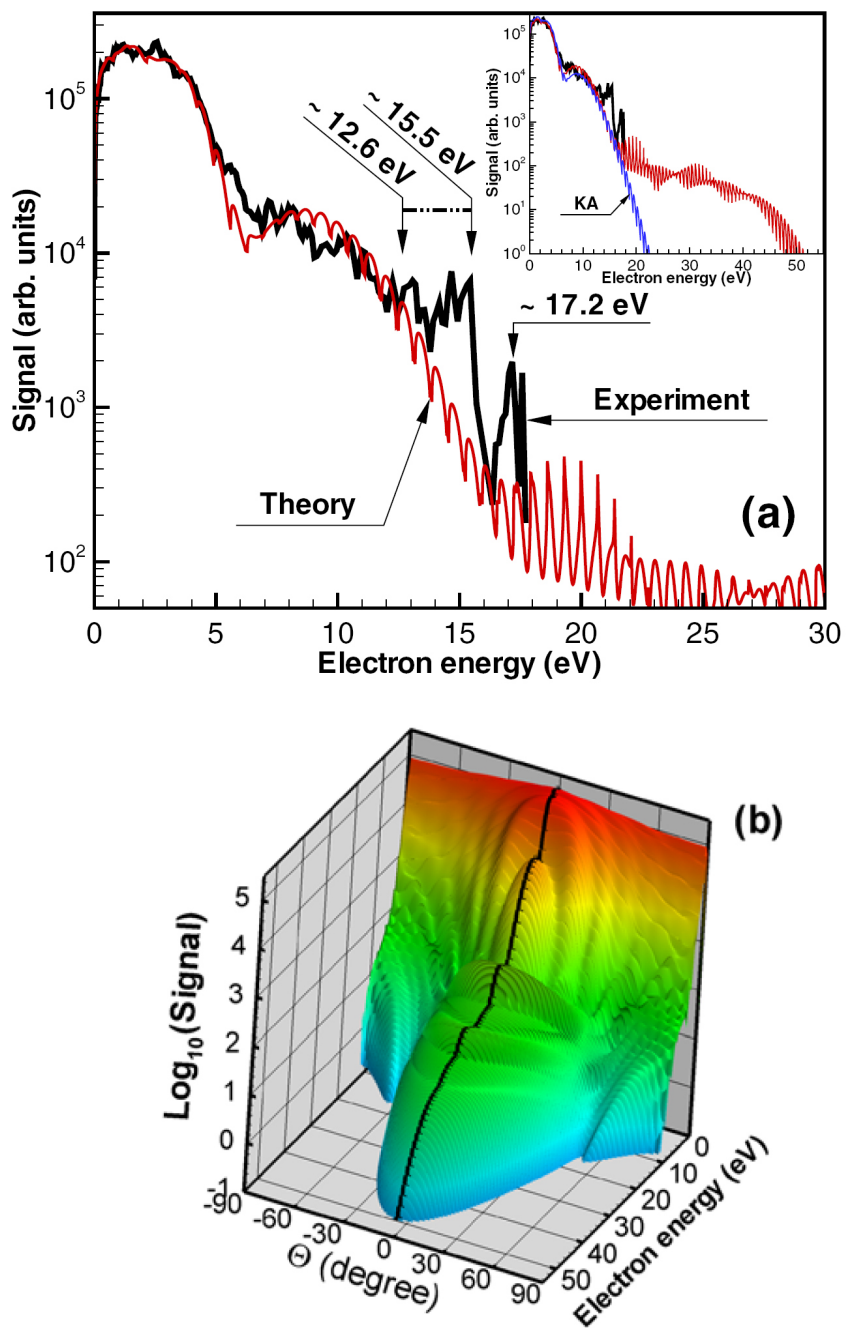

Figure 2. (a) Theoretical (RA) (thin solid curve) and experimental (thick solid curve) ATD spectra of $\mathrm{F}^{-}$for $\omega=$ $0.2026(\lambda=1.8 \mu \mathrm{m})$ and $\theta=0$ as a function of electron energy. The inset extends the theoretical RA predictions to higher electron energies and compares them to the KA result. The theoretical results include focal averaging for a peak laser intensity of $I=1.6 \times I_{\exp }=1.76 \times 10^{13} \mathrm{~W} \mathrm{~cm}^{-2}(F=0.34)$. (b) Focal-averaged 3D angular distribution for the same parameters as in panel (a).

experimental electron yield results of Kiyan and Helm [8] on intense field detachment of $\mathrm{F}^{-}$. We find excellent agreement with the experimental results in the low energy region of the spectrum after taking into account focal averaging and optimizing the peak laser intensity. We also find that the highest energy experimental results lie just at the beginning of our predicted rescattering plateau. Discrepancies between our essentially exact numerical predictions for an effective one-electron system at the highest energies considered experimentally (i.e., just below our predicted rescattering plateau) are attributed to two-electron excitations that are known from other theoretical and experimental investigations.

Note added in article proof: We have become aware of an alternative theoretical analysis of the $\mathrm{F}^{-}$ data by D. B. Milosevic et al., presented in Reference [22]. 
Acknowledgments

We thank P. Burrow, I. Fabrikant, G. Gallup, and A. Khuskivadze for fruitful discussions about atomic-negative-ion resonances and I. Kiyan for providing us with the experimental data. This work was supported in part by NSF Grant PHY-0070980, by the Joint Grant VZ-010-0 of the CRDF and the RF Ministry of Education, and by the University of Nebraska-Lincoln Research Computing Facility.

\section{References}

[1] Keldysh L V 1964 Zh. Eksp. Teor. Fiz. 47 1945; Keldysh L V 1965 Sov. Phys. - JETP 201307 (Engl. Transl.)

[2] Lohr A, Kleber M, Kopold R and Becker W 1997 Phys. Rev. A 55 R4003

[3] Becker W, Grasbon F, Kopold R, Milosevic D B, Paulus G G and Walther H 2002 Adv. At. Mol. Opt. Phys. 4835

[4] Manakov N L and Fainshtein A G 1980 Zh. Exp. Teor. Fiz. 79 751; Manakov N L and Fainshtein A G 1980 Sov Phys. - JETP 52382 (Engl. Transl.)

[5] Borca B, Frolov M V, Manakov N L and Starace A F 2001 Phys. Rev. Lett. 87 133001; Borca B, Frolov M V, Manakov N L and Starace A F 2002 Phys. Rev. Lett. 88193001

[6] Manakov N L, Frolov M V, Borca B and Starace A F 2003 J. Phys. B: At. Mol. Opt. Phys. 36 R49

[7] Frolov M V, Manakov N L, Pronin E A and Starace A F 2003 Phys. Rev. Lett. 91053003

[8] Kiyan I Yu and Helm H 2003 Phys. Rev. Lett. 90183001

[9] Gribakin G F and Kuchiev M Yu 1997 Phys. Rev. A 553760

[10] Demkov Yu N and Drukarev G F 1981 Zh. Eksp. Teor. Fiz. 81 1218; Demkov Yu N and Drukarev G F 1981 Sov Phys. - JETP 54650 (Engl. Transl.)

[11] Andreev S P, Karnakov B M, Mur V D and Polunin V A 1984 Zh. Eksp. Teor. Fiz. 86 866; Andreev S P, Karnakov B M, Mur V D and Polunin V A 1984 Sov. Phys. - JETP 59506 (Engl. Transl.)

[12] Landau L D and Lifshitz E M 1992 Quantum Mechanics 4th edn (Oxford: Pergamon)

[13] Baz' A I, Zel'dovich Ya B and Perelomov A M 1971 Scattering, Reactions and Decays in Nonrelativistic Quantum Mechanics 2nd edn (Moscow: Nauka)

[14] Nikishov A I and Ritus V I 1964 Zh. Eksp. Teor. Fiz. 19 529; Nikishov A I and Ritus V I 1964 Sov Phys. JETP 46776 (Engl. Transl.)

[15] Ohmura T and Ohmura H 1960 Phys. Rev. A 118154

[16] Walker B, Sheehy B, Kulander K C and DiMauro L F 1996 Phys. Rev. Lett. 775031

[17] Nandor M J, Walker M A and Van Woerkom L D 1998 J. Phys. B: At. Mol. Opt. Phys. 314617

[18] Becker A, Plaja L, Moreno P, Nurhuda M and Faisal F H M 2001 Phys. Rev. A 64023408

[19] NIST Atomic Spectra Database Data http://physics.nist.gov

[20] Golovinskii P A 1993 Opt. Spektrosk. 74 647; Golovinskii P A 1993 Opt. Spectrosc. (USSR) 74386 (Engl. Transl.)

[21] Buckman S J and Clark C W 1994 Rev. Mod. Phys. 66539

[22] Milosevic D B, Gazibegovic-Busuladzic A, and Becker W 2003 Phys. Rev. A at press 